\title{
Research progress of circular RNA in digestive tract tumors: a narrative review
}

\author{
Haojia Wang $^{1 "}$, Yanghui Xiang ${ }^{1 \#}$, Rui Hu ${ }^{1 \#}$, Rui Ji ${ }^{2,3}$, Yuping Wang ${ }^{2,3}$ \\ ${ }^{1}$ The First School of Clinical Medicine, Lanzhou University, Lanzhou, China; ${ }^{2}$ Department of Gastroenterology, The First Hospital of Lanzhou University, \\ Lanzhou, China; ${ }^{3}$ Key Laboratory for Gastrointestinal Diseases of Gansu Province, The First Hospital of Lanzhou University, Lanzhou, China \\ Contributions: (I) Conception and design: Y Wang; (II) Administrative support: R Ji; (III) Provision of study materials or patients: R Ji; (IV) Collection \\ and assembly of data: H Wang; (V) Data analysis and interpretation: None; (VI) Manuscript writing: All authors; (VII) Final approval of manuscript: \\ All authors. \\ \#These authors contributed equally to this work. \\ Correspondence to: Yuping Wang. Department of Gastroenterology, Key Laboratory for Gastrointestinal Diseases of Gansu Province, The First \\ Hospital of Lanzhou University, No. 1 West Donggang Road, Lanzhou 730000, China. Email: wangyuping@lzu.edu.cn.
}

\begin{abstract}
Circular RNA (circRNA) is a newly discovered non-coding RNA with a closed loop structure. The 3 ' end and the 5' end of circRNA are covalently bonded and not easily degraded by nucleases. Besides, circRNA can exist in eukaryotes widely and conservatively, and it is stably expressed in human liver, brain, lung and other organs. CircRNA can also regulate gene expression by acting on miRNA just like "miRNA sponge". With the deepening of research, growing literature has evidence shows that circRNA is related to tumors closely. For tumors, a large part of malignant ones are gastrointestinal tumors (GIST). In recent years, there are some researches which show that circRNA plays an important role in the occurrence and development, detection and treatment, and prognostic evaluation of malignant tumors of the digestive system. This article summarizes the literature reports on circRNA and GIST since March 2019. From 326 records sc reened, 79 full text articles were assessed for eligibility and 29 studies met the inclusion criteria. We reviewed the relationship between circRNA and GIST, and its role in the development of tumors. These studies show that in the future, circRNA has the potential to be used as a molecular marker for the detection and treatment of GIST.
\end{abstract}

Keywords: Tumor; circular RNA (circRNA); research progress

Submitted Aug 21, 2020. Accepted for publication Nov 06, 2020.

doi: $10.21037 /$ tcr-20-2708

View this article at: http://dx.doi.org/10.21037/tcr-20-2708

\section{Introduction}

In 1976, Sanger's (1) team discovered a viroid with a ring structure when they were making a research about higher plants. They named it circular RNA (circRNA). In 2012, through a systematic research, Salzman (2) discovered that circRNA is a kind of non-coding RNA (ncRNA) with a circular structure. Its $3^{\prime}$ end and 5' end are covalently bound, and it does not have a $3^{\prime}$ cap structure and a $5^{\prime}$ polyadenylic acid tail, which means that it is not sensitive to nucleases. Besides, circRNA is ubiquitous in eukaryotes, and it is stably expressed in human livers, brains, lungs, and other organs (3-5). Some studies have shown that circRNA can affect the expression of microRNA (miRNA) in cells through the "miRNA sponge" (6) effect, and it can regulate gene expression through the relationship of circRNA $\rightarrow$ miRNA $\rightarrow$ mRNA as well. In recent years, with the development of bioinformatics technology and highthroughput chip technology, growing literature has shown that circRNA plays an important role in the occurrence and development of tumors.

Compared to the $19^{\text {th }}$ century and before, the spectrum of diseases and deaths has undergone significant changes 
since the $20^{\text {th }}$ century: infectious diseases that have affected human health for thousands of years have gradually been brought under control since the discovery of antibiotics. However, a gradual increasing incidence of chronic and non-communicable diseases have posed another threat to human beings. In the 21 st century, the incidence and mortality of malignant tumors have increased year by year. Especially in China, the mortality rate of malignant tumors is even higher than the global average. According to some estimates, by 2030, the death rate of malignant tumors will rise to 118 per 100,000, which is higher than other diseases except heart disease (7). As a developing country, both the mortality and the morbidity of malignant tumors in China are higher than the global average. Besides, the proportion of gastrointestinal tumors (GIST) in China is increasing even year by year. In 2009, Chen et al. (8) analyzed 104 tumor registries nationwide and found that the esophagus cancer, gastric cancer, liver cancer, and colorectal cancer constitute $40.75 \%$ of the top 10 malignant tumors. This does cause a great social burden. However, in recent years, with the development of molecular detection technology, finding effective and non-invasive molecular targets has become a new idea for early diagnosis, targeted therapy, and prognostic analysis of GIST. Luckily, because of its wide existence and stability, circRNA might be a new target for inspection and treatment of digestive tract tumors. We present the following article in accordance with the Narrative Review reporting checklist (available at http:// dx.doi.org/10.21037/tcr-20-2708).

\section{Methods}

This study retrieved and screened articles related to circRNA included in databases such as PubMed and CNKI since March 2019. The search formula uses "circular RNA" and "circRNA" as keywords related to circRNA. Since there are many types of malignant tumors in the digestive tract and the names of tumors with different degrees of differentiation are different, in order to prevent omissions, this study searches for tumors and circRNArelated literature, and the digestive tract-related articles that meet the requirements are screened by reading abstracts. A total of 320 tumor-related circRNA literature were initially screened since March 2019, and 6 were manually searched for a total of 326 literature. After excluding other systemic malignancies, 79 literature related to GIST were obtained.

For the preliminary screening of the literature, rescreen according to the following inclusion and exclusion criteria. Inclusion criteria: (I) studies related to malignant tumors of the digestive tract; (II) the malignant tumors were confirmed pathologically; (III) languages were Chinese or English; (IV) those with higher impact factors were selected for repeated site studies. Exclusion criteria: (I) no experiment or only cytology experiment; (II) review and analysis literature; (III) conference papers, abstracts and lectures. After intensive reading and screening, nine articles related to gastric cancer, eight articles related to colorectal cancer, two articles related to esophageal cancer, three articles related to pancreatic cancer, seven articles related to liver cancer were selected, a total of 29 articles

\section{Overview of circRNA}

\section{The discovery of circRNA}

Of the known human genome, coding RNA (mRNA) that encodes functional proteins only accounts for about $1 \%$ of the RNA system (9), and the rest that does not perform translation functions directly are called ncRNAs. In recent years, with the deepening of ncRNA research, more and more evidence shows that ncRNA can regulate gene expression and produce corresponding biological functions. It has been proved that miRNA, long non-coding RNA (lncRNA) and some other RNA is related to the occurrence, development, diagnosis, and treatment of many diseases closely. With the development of RNA sequencing (RNA-seq), circRNA, one kind of circular ncRNA that does not have a 3' cap structure and a 5' polyadenylic acid tail, has been noticed gradually. CircRNA was first observed by Sanger (1). Later, some studies found that circRNA is widespread in eukaryotes. Jeck and others (10) detected more than 25,000 kinds of circRNA expressions in human fibroblasts with the help of RNA-seq technology. Besides, Memczak et al. (11) detected 1,950 kinds of human circRNA, 1,903 kinds of mouse circRNA, and 724 kinds of nematode circRNA. In the beginning, many scholars believed that circRNA is just a non-functional misplacing or a by-product in the splicing process (12), until Salzman (2) made a systematic research showing that circRNA can regulate gene expression in 2012. In his subsequent studies, Salzman went a further step, which confirmed that circRNA is indeed related to the occurrence of diseases closely.

\section{The production process of circRNA}

Post-transcriptional processing is a necessary step in the 


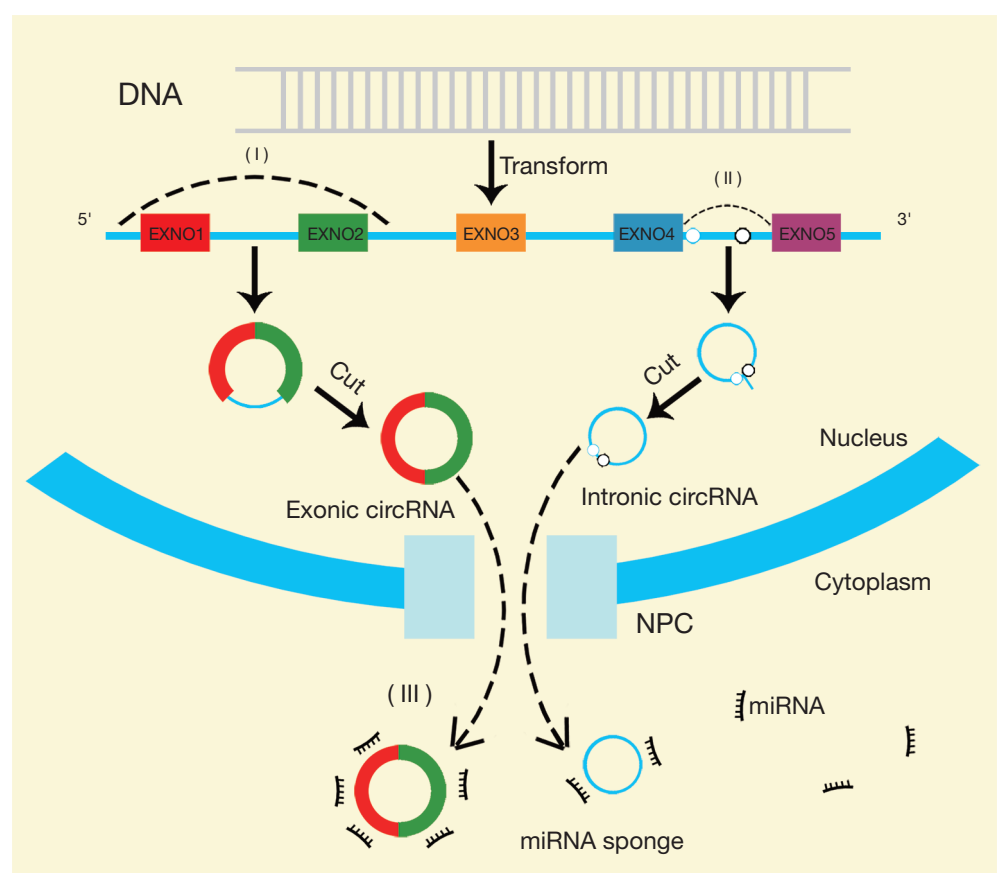

Figure 1 CircRNA production mechanism. (I) After exons are circularized, the introns are cut out to form mature circRNA. (II) Introns drive circularization and pruning out redundant introns to complete maturation. (III) Mature circRNA is transported to the cytoplasm through the nuclear pore complex and exerts its miRNA sponge function. CircRNA, circular RNA; NPC, nuclear pore complex.

process of RNA maturation. Moreover, alternative splicing, as a processing method, plays an important role in the formation of circRNA. As for circRNA, according to its source, it can be divided into three categories: circRNA derived from exons, circRNA derived from introns, and circRNA derived from both exons and introns. First, for exon-derived circRNA, Lariat-driven circularization and exon skipping are the main principles for the generation of it. For maturation of exon-derived circRNA, the 3' end of the exon-derived splice donor and the splice acceptor 5 ' end would be covalently combined to form a ring structure, and then it would cut out intron from itself. Second, Intronderived circRNA is often matured in long flanking introns containing Alu repetitive sequences: It would be selfcircularized through Alu complementation, and then other redundant introns would be removed (Figure 1). No matter how it is derived, mature circRNA can be transported to the cytoplasm through the nuclear pore complex (NPC), and then it would make corresponding biological effects in the cytoplasm (13).

Due to its special structure, circRNA has the following characteristics: (I) the structure of circRNA is stable. The head and the tail of circRNA are covalently combined to form a ring structure, which cannot be easily degraded by nucleases (14); (II) most of them are located in the cytoplasm, while only a few located in the nucleus (15); (III) most of them belong to ncRNA; (IV) most of them are composed of exons, while the rest is composed of either introns or exons and introns; $(\mathrm{V})$ there is one kind of variable circularization for circRNA, which means that a gene can produce many different kinds of circRNA (6); (VI) most of them are evolutionarily conserved $(6,10)$; (VII) there are some kinds of specificity of tissue and time in the body distribution of circRNA; ()VIII circRNA is fairly rich in both variety and numbers, and the number of some circRNA can even be 10 times as many as their corresponding linear RNAs (15); (IX) it contains miRNA response primitives, which can interact with miRNAs to regulate gene expression at the transcriptional or post-transcriptional level (6).

\section{CircRNA regulates gene expression through miRNA sponge action}

Most current researches on circRNA shows that it can bind to the corresponding miRNA and inhibit its biological activity through a specific way that is similar to "miRNA 
sponge" effect. That's why circRNA can regulate the expression of downstream genes. In 2011, for the first time in history, Hansen and others (16) discovered that cerebellar degeneration-related protein 1 (CDR1) can produce a circular transcription product, antisense to the CDR1 transcript (CDR1as). In addition, some studies have shown that CDR1as contains more than $70 \mathrm{miR}-7$ response elements (MRE) (6), but it will not be degraded and inactivated by miR-7, which means that there are some kinds of specific interactions between CDR1as and miR-7. Subsequent studies have shown that the expression of miR7 will decrease when CDR1as is overexpressed, while it will be higher than normal when CDR1as is knocked down, which leads to a decrease in the expression of miR-7 targets. This phenomenon indicates that CDR1as can inhibit the activity of miR-7 so that it can regulate the corresponding signal pathway. In the experiment of zebrafish embryos, the embryonic midbrain volume decreased after the overexpression of CDR1as, and accordingly, the midbrain volume and morphology were restored after miR-7 precursor administration (11). That was consistent with the phenotype produced by knocking down miR-7 and further confirmed the above hypothesis. In addition, the sex-determining region $\mathrm{Y}$ (SRY) of the $\mathrm{Y}$ chromosome can also be transcribed to produce a circRNA, and the transcribed RNA will contain 16 miR-138 MREs just like CDR1as. The circRNA can also bind miR-138 and inhibit its biological activity, thereby affecting the expression of downstream target genes (6). These two experiments above have proved that circRNA can regulate gene expression in vivo by affecting miRNA levels.

\section{The relationship between circRNA and GIST}

With the development of technology and the deepening of circRNA research, more and more studies have shown that circRNA plays an important role in the occurrence, development, and progression of various malignant tumors. Some literature shows that, for common GIST such as gastric cancer, liver cancer, esophageal cancer, and colorectal cancer, the expression of a variety of circRNAs is significantly different from that of normal tissues. Besides, its expression level is related to tumor cell migration, invasion and Behavioral abilities such as proliferation closely. In addition, there are many differences among expressions in tumor cells of different levels of differentiation. This article will briefly review the relationship between circRNA and digestive tract tumors in some selected literature.

\section{CircRNA and esophageal cancer}

Esophageal cancer is one kind of malignant tumor that originates from the esophageal epithelium. It is common worldwide. It ranks third in incidence and fourth in death among malignant tumors in China. A large number of studies have shown that a variety of circRNAs are associated with esophageal cancer tissues, and there are obvious differential expressions of circRNA between those cancer tissues and normal tissues. Shi (17) found that the expression of circ_0030162 in esophageal squamous cell carcinoma was significantly lower than that in paracancerous tissues. His study, which is focused on 69 pairs of esophageal squamous cell carcinoma patients and normal patient tissue samples, showed that the expressions of circ_0063772 both in their tissues and plasma are different. The results show that circ_0063772 has the potential as a diagnostic marker, and when combined with it in plasma and tissue as a diagnostic marker, its effect is better (sensitivity $81.81 \%$, specificity $65.91 \%, 95 \%$ CI: $0.070-0.890)$. And later experiments found that the overexpression of the circRNA can significantly inhibit the proliferation, invasion, and migration of cancer cells and other behavioral functions. Also, some scholars have found that the expression of circ_0067934 (18) and circ-PRKCI (19) is significantly up-regulated in esophageal cancer, and the up-regulation of the latter can promote the proliferation and invasion of esophageal squamous cell carcinoma and esophageal adenocarcinoma. Besides, Shi et al. (19), through immunoprecipitation assay and luciferase assay, found that circRNA can promote the progression of esophageal cancer by affecting the miR-3680-3p/ART-3 signaling pathway.

\section{CircRNA and liver cancer}

Liver cancer is a common malignant tumor worldwide. It originates from hepatocytes or bile duct epithelial cells. It is clinically divided into primary hepatocellular carcinoma (HCC) and intrahepatic cholangiocarcinoma (ICC). Among them, HCC is the most common, and it accounts for more than $90 \%$ of malignant tumors. The occurrence of liver cancer is related to many factors. The common pathogenic factors include hepatitis virus infection and long-term alcoholism. The early symptoms of liver cancer are not obvious, so it is difficult to be found. Common examination methods of it include imaging examination and serum alpha-fetoprotein (AFP) measurement, but they both have certain limitations. However, some scholars have found 
that for some HCCs with insignificant AFP elevation, early detection can be performed by the determination of circRNA. Studies such as Li et al.'s (20) have shown that circSMARCA5 has a certain diagnostic value for HCC with AFP $<200 \mathrm{ng} / \mathrm{mL}$. Especially for the detection of circSMARCA5 in the plasma of patients, high accuracy can be obtained. The expression of circRNA also has a certain influence and significance on the progression of liver cancer. The expression of circ-BIRC6 in HCC patients was significantly higher than that in the control group. The study showed that it was co-localized with miR-3918 in the cells. After the cells were silenced, the expression of miR-3918 increased, while the behavioral ability of HCC cell proliferation and invasion decreased. Besides, Cox regression analysis showed that the survival cycle of patients with high expression of circ-BIRC6 was much lower than that of patients with low expression (21). The above studies indicate that circRNA can affect the progression of HCC as well as the life cycle of HCC patients. Traditional liver cancer treatment includes surgical treatment and drug treatment. However, with the deepening of research between circRNA and liver cancer, the signaling pathways supported by circRNA and its influence on patient prognosis provide new ideas for HCC treatment. HCC has certain immune tolerance, so the effect of drug treatment is often not ideal. Luckily, Huang et al. (22) found that circMET can promote HCC immune tolerance through the miR-30-5p/Snail/DPP4/CXCL10 axis. During the experiment, the inhibitor sitagliptin can inhibit immune tolerance and improve the therapeutic effect by giving DPP4, which suggests that circRNA can be used as a breakthrough in the treatment of liver cancer. In addition, the latest research has found that there are some exogenous circRNAs in HCC cells, such as circ_0051443 (23), which is transferred from normal cells into HCC cells in the form of exosomes and binds to miR-311-3p to promote the expression of BAK1. That's the case that can delay cell cycle and promote cell apoptosis.

\section{CircRNA and colorectal cancer}

Colorectal cancer is one kind of common malignant tumor of the digestive system. It is classified according to the location of its occurrence. In recent years, the prevalence of colorectal cancer in China has increased significantly, especially in large cities. Besides, it is pretty common among people aged 41-65 years. The incidence of colorectal cancer is related to genetics, age, obesity, eating habits and other factors. Due to the lack of specificity in its early clinical manifestations, the overall prognosis and quality of life of patients are poor. Nevertheless, some recent studies have shown that a variety of circRNAs are differentially expressed in samples of patients with colorectal cancer, and have the potential to be used as molecular markers for disease diagnosis: Circ_0008285, circ-FBXW7, and many other kinds of circRNA can affect tumor progression and malignant behavior $(24,25)$. Lu and some other researchers (25) constructed a mouse model. They found that the tumor volume in the circ-FBXW7 overexpression group was significantly lower than that in the control group, while the gene silencing group showed the opposite trend. These indicate that circRNA can have an impact on the occurrence and development of colorectal cancer. Besides, new blood vessels play an important role in the progression of colorectal cancer. Some scholars have shown that the circ_001971/miR-29c-3p axis can regulate the production of tumor neovascularization by affecting the downstream target vascular endothelial growth factor A (VEGFA). When the expression of circ_001971 is low, angiogenesis is significantly reduced, and the tumor growth rate is also significantly slowed. Also, its expression in different TNM stages is different. All above shows one idea: it has the potential to be used as a molecular therapy target and evaluation staging. This year, with the continuous deepening of research on circRNA and tumors, more and more evidence shows that the diagnosis, treatment and prognosis evaluation of colorectal cancer can be affected by the expression of a variety of circRNAs. For instances, circ_0136666 (26) can be used as a miR-136 sponge to promote the expression of the downstream target SH2B1, thereby inhibiting tumor cell proliferation and invasion. Besides, survival analysis showed that the survival rate of patients with high expression of circRNA was significantly higher than that of the low expression group, suggesting that this site is different from direct expression, and is relevant to the prognosis of colon cancer patients, and it can also be used as a targeted marker to guide treatment. In addition, when studying $\operatorname{circFNDC3B}$, Pan et al. (27) found that it encodes a new protein circFNDC3B-218aa, and up-regulating the expression of this protein showed significant inhibition of the progression of colon cancer. All those suggest that we have a signal pathway mediated by circRNA. It can be used as a potential target for tumor molecular therapy, and its upstream and downstream sites have the possibility of being used as a therapy-oriented molecular marker. 


\section{CircRNA and gastric cancer}

Gastric cancer is one kind of malignant tumor with a high incidence worldwide and one of the main causes of death. As a country with large numbers of gastric cancer, China has kept the incidence and mortality of gastric cancer at a high level for many years. Especially in Wuwei, Gansu, these two numbers are even much higher than national and world average levels. What's a little better is that, in recent years, with the development of molecular technology, the selection of effective and non-invasive molecular targets has become a new hot spot for early detection, targeted therapy and prognosis evaluation of gastric cancer. Chang and others (28) analyzed the GEO database and found that there is a wide range of circRNA differential expressions between gastric cancer tissues and normal tissues. Further researches on its function and signaling pathways showed that differentially expressed circRNAs can regulate miRNA expression and downstream signaling pathways. Playing a cancer-promoting or anti-tumor effect, circRNA can be confirmed that it plays an important role in the occurrence and development of gastric cancer. Besides, some current

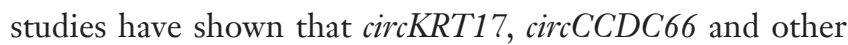
circRNAs are significantly overexpressed in gastric cancer tissues and cells. For instance, CCK- 8 and Transwell tests have shown that their high expression levels can promote the proliferation, invasion and migration of gastric cancer cells and other malignant behaviors. These results have been confirmed in mouse subcutaneous tumor formation experiments $(29,30)$. With the deepening of exploration, researchers found that the effect of circRNA on gastric cancer may be due to its sponge-like binding effect on miRNA, which affects the relevant signal pathways. Besides, Xie et al. analyzed the miRDB database and found that after knocking down the expression of circ_VANGL1, the expression levels of miR-605-3p, miR-377-5p, miR-4468 and $\mathrm{miR}-5008-5 \mathrm{p}$ were all reduced (31). In addition to others studies, it has been shown that circRNA can affect gastric cancer cells through multiple signal axes such as miR-6792-3p/CAV1 and miR-6792-3p/CAV1.

The lack of early detection methods for gastric cancer and unsatisfactory treatment effects are the main reasons for the low overall survival rate and disease-free survival rate of gastric cancer patients. Digestive endoscopy plus pathological testing has always been the recognized correct standard for the diagnosis of gastric cancer, but it is not a routine-laboratory test. In addition, compared with blood tumor markers, the detection method is more difficult.
What's worse is that there is not enough specificity in the early clinical manifestations of gastric cancer. Therefore, early detection has always been difficult. $\mathrm{Lu}$ and others (32) studied 30 patients with gastric cancer and found that circRanGAP1 is highly expressed in plasma samples of gastric cancer patients. This result suggests that circRNA can be used as a biomarker for gastric cancer detection. Besides, Liu and others (33) found that circ_0008797 is sensitive to cisplatin, namely the expression in cell lines is significantly higher than that in cisplatin-resistant cell lines. Also, overexpression of circ_0008797 can increase the sensitivity of gastric cancer cells to cisplatin, which suggests that circRNA can be used as a new entry point for gastric cancer combined therapy.

\section{CircRNA and pancreatic cancer}

The vast majority of pancreatic cancer originates from pancreatic duct epithelial cells. Due to the concealment of early manifestations and difficult methods of examination, most patients are already in the middle or even the late stages of the disease when they are discovered, which really makes pancreatic cancer one of the malignant tumors with the lowest survival rate. Li et al. (34) used RNAseq technology to detect 453 circRNAs with significant differences among expressions in samples from eight pancreatic cancer patients, and found that their expression levels have a certain degree of relationship with tumor progression and malignant behavior. Guo et al. (35) studied 208 patients with pancreatic ductal adenocarcinoma (PDAC) samples and found that circBFAR is overexpressed in tumor tissues, and proved that the circRNA can promote mesenchyme by binding miR-34b-5p. According to Guo et al., the expression of mesenchymal-to-epithelial transition factor (MET) would further activate downstream Akt phosphorylation and promote the proliferation and invasion of PDAC through the MET/PI3K/Akt axis. Besides, gemcitabine (GEM) is an important chemotherapeutic drug for pancreatic cancer, but some studies have shown that some pancreatic cancer patients can develop resistance to GEM, which makes the treatment effect unsatisfactory. Liu et al. (36) found that circHIPK3 is highly expressed in pancreatic cancer cells, and knocking down circHIPK3 can inhibit GEM drug resistance of tumor cells, and further confirmed that the above performance is due to its binding to miR-330-5p that causes the overexpression of downstream RASSF1. A large number of studies have confirmed that the abnormal expression of circRNA 
affects the progression and prognosis of pancreatic cancer tumors, and has a certain guiding role in early diagnosis and targeted therapy. Table 1 briefly summarizes the circRNAs involved in the selected documents in this article, as well as their signal pathways and biological functions (Table 1).

\section{Discussion}

CircRNA has been considered as the product of splicing errors for a long time since its discovery. Luckily, in recent years, as its expression and function have been clarified, circRNA has quickly become a hotspot of research in ncRNA. As more and more circRNAs are discovered and studied, their roles in diseases have gradually attracted more and more attention. Here comes a good case. Tumors of the digestive tract are one of the main causes of death. For a long time, the early diagnosis and treatment effects of it are not ideal at all, under which condition it has become a general trend to find effective molecular target sites. Under this background, the relationship between circRNA and digestive tract tumors has become a hotspot of research. Some current studies have confirmed that a large number of circRNAs are related to the growth, invasion and TNM staging of gastric cancer, liver cancer, colorectal cancer and other malignant tumors. Some circRNA sites that are highly expressed in plasma are also expected to be used for early detection. In addition, circRNA has also been proven to play a role in the treatment of malignant tumors, and it also provides new ideas for future tumor combination therapy to improve the efficacy of treatment by drugs. It is relatively regrettable that the research of circRNA on tumor occurrence and development is still at the experimental stage, and the experimental results obtained have not yet been applied to the clinical front line. However, it is believed that with the continuous elucidation of the mechanism of its influence and the further improvement of technical means, circRNA could be more beneficial for diagnosis and treatment of digestive tract tumors.

Table 1 CircRNAs related to gastrointestinal tumors

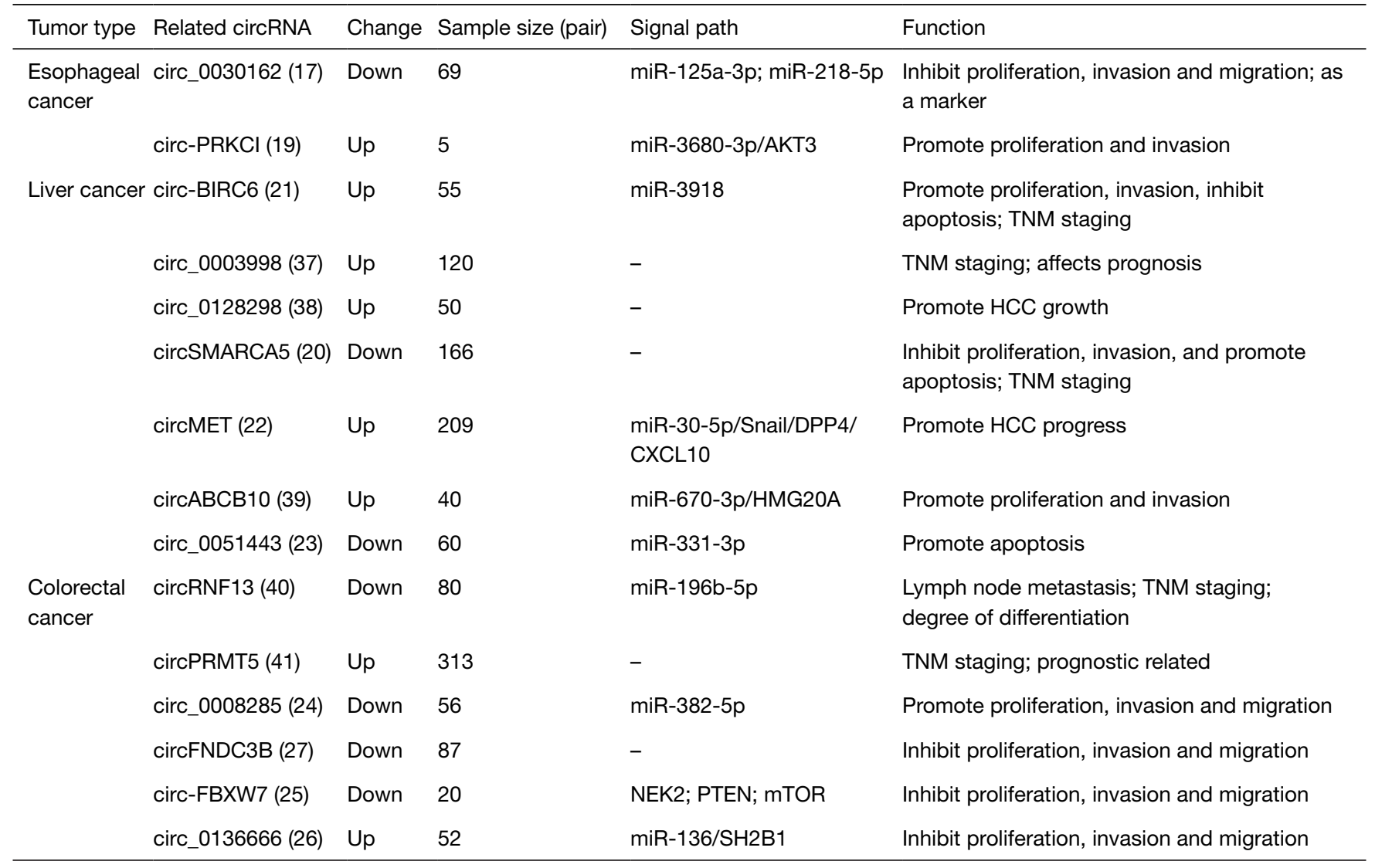

Table 1 (continued) 
Table 1 (continued)

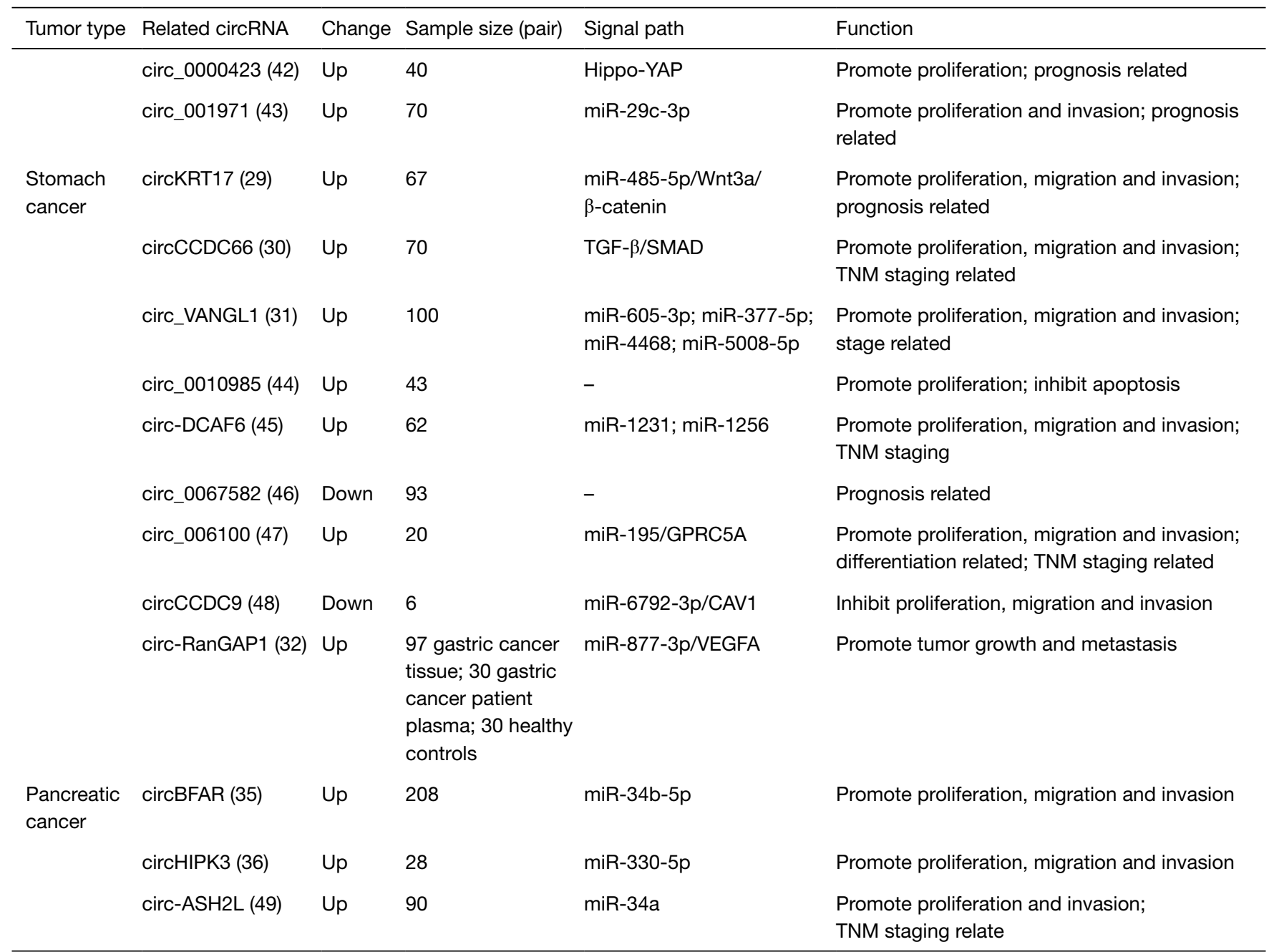

CircRNA, circular RNA; HCC, hepatocellular carcinoma.

\section{Acknowledgments}

Funding: None.

\section{Footnote}

Reporting Checklist: The authors have completed the Narrative Review reporting checklist. Available at http:// dx.doi.org/10.21037/tcr-20-2708

Conflicts of Interest: All authors have completed the ICMJE uniform disclosure form (available at http://dx.doi. org/10.21037/tcr-20-2708). The authors have no conflicts of interest to declare.
Ethical Statement: The authors are accountable for all aspects of the work in ensuring that questions related to the accuracy or integrity of any part of the work are appropriately investigated and resolved.

Open Access Statement: This is an Open Access article distributed in accordance with the Creative Commons Attribution-NonCommercial-NoDerivs 4.0 International License (CC BY-NC-ND 4.0), which permits the noncommercial replication and distribution of the article with the strict proviso that no changes or edits are made and the original work is properly cited (including links to both the formal publication through the relevant DOI and the 
license). See: https://creativecommons.org/licenses/by-nc$\mathrm{nd} / 4.0 /$.

\section{References}

1. Sanger HL, Klotz G, Riesner D, et al. Viroids are SingleStranded Covalently Closed Circular RNA Molecules Existing as Highly Base-Paired Rod-Like Structures. Proc Natl Acad Sci U S A 1976;73:3852-56.

2. Salzman J, Gawad C, Wang PL, et al. Circular RNAs are the predominant transcript isoform from hundreds of human genes in diverse cell types. PLoS One 2012;7:e30733.

3. Chen LJ, Nan AR, Zhang N, et al. The aberrant expression of circular RNA 001988 and its target genes in benzo [a] pyrene-induced lung cancer. Journal of Environment and Health 2016;33:565-8.

4. Rybak-Wolf A, Stottmeister C, Glažar P, et al. Circular RNAs in the Mammalian Brain Are Highly Abundant, Conserved, and Dynamically Expressed. Mol Cell 2015;58:870-85.

5. Zhong Z, Lv M, Chen J. Screening differential circular RNA expression profiles reveals the regulatory role of circTCF25-miR-103a-3p/miR-107-CDK6 pathway in bladder carcinoma. Sci Rep 2016;6:30919.

6. Hansen TB, Jensen TI, Clausen BH, et al. Natural RNA circles function as efficient microRNA sponges. Nature 2013;495:384-8.

7. Zhou YR. Epidemic Trend and Control for Cancer Mortality. China Cancer 2011;20:256-8.

8. Chen WQ, Zhang SW, Zheng RS, et al. Report of Cancer Incidence and Mortality in China, 2009. Chin J Cancer Res 2013;25:10-21.

9. Venter JC, Adams MD, Myers EW, et al. The sequence of the human genome. Science 2001;291:1304-51.

10. Jeck WR, Sorrentino JA, Wang K, et al. Circular RNAs are abundant, conserved, and associated with ALU repeats. RNA 2013;19:141-57.

11. Memczak S, Jens M, Elefsinioti A, et al. Circular RNAs are a large class of animal RNAs with regulatory potency. Nature 2013;495:333-8.

12. Cocquerelle C, Mascrez B, Hétuin D, et al. Mis-splicing yields circular RNA molecules. FASEB J 1993;7:155-60.

13. Li J, Yang J, Zhou P, et al. Circular RNAs in cancer: novel insights into origins, properties, functions and implications. Am J Cancer Res 2015;5:472-80.

14. Zhang XO, Wang HB, Zhang Y, et al. Complementary sequence-mediated exon circularization. Cell
2014;159:134-47.

15. Lasda E, Parker R. Circular RNAs: diversity of form and function. RNA 2014;20:1829-42.

16. Hansen TB, Wiklund ED, Bramsen JB, et al. miRNAdependent gene silencing involving Ago2-mediated cleavage of a circular antisense RNA. EMBO J 2011;30:4414-22.

17. Shi P. Screening for and functional analysis of epigenetic biomarkers of circRNAs in esophageal squamous cell carcinoma [Master]. Nanjing Medical University, 2019.

18. Wang S, Li J, Liang $\mathrm{Y}$, et al. A systematic review of circRNAs in digestive system malignancies. Journal of Practical Oncology 2018;33:334-9.

19. Shi N, Shan B, Gu B, et al. Circular RNA circPRKCI functions as a competitive endogenous RNA to regulate AKT3 expression by sponging miR-3680-3p in esophageal squamous cell carcinoma. J Cell Biochem 2019;120:10021-30.

20. Li Z, Zhou Y, Yang G, et al. Using circular RNA SMARCA5 as a potential novel biomarker for hepatocellular carcinoma. Clin Chim Acta 2019;492:37-44.

21. Yang G. circ-BIRC6 promotes hepatocellular carcinoma progression by targeting the miR-3918/Bcl2 axis [Master]. Shandong University, 2019.

22. Huang XY, Zhang PF, Wei CY, et al. Circular RNA circMET drives immunosuppression and anti-PD1 therapy resistance in hepatocellular carcinoma via the miR-30-5p/ snail/DPP4 axis. Mol Cancer 2020;19:92.

23. Chen W, Quan Y, Fan S, et al. Exosome-transmitted circular RNA hsa_circ_0051443 suppresses hepatocellular carcinoma progression. Cancer Lett 2020;475:119-28.

24. Wang J, Luo J, Liu G, et al. Circular RNA hsa_ circ_0008285 inhibits colorectal cancer cell proliferation and migration via the miR-382-5p/PTEN axis. Biochem Biophys Res Commun 2020;527:503-10.

25. Lu H, Yao B, Wen X, et al. FBXW7 circular RNA regulates proliferation, migration and invasion of colorectal carcinoma through NEK2, mTOR, and PTEN signaling pathways in vitro and in vivo. BMC Cancer 2019;19:918.

26. Jin C, Wang A, Liu L, et al. Hsa_circ_0136666 promotes the proliferation and invasion of colorectal cancer through miR-136/SH2B1 axis. J Cell Physiol 2019;234:7247-56.

27. Pan Z, Cai J, Lin J, et al. A novel protein encoded by circFNDC3B inhibits tumor progression and EMT through regulating Snail in colon cancer. Mol Cancer 2020;19:71.

28. Chang XS, Han T, Kang ZC, et al. Differential expression 
of circular RNA and its function and pathway involved in gastric cancer based on GEO database. Clinical Journal of Medical Officers 2019;47:1298-302.

29. Zheng R, Zhang X, Nie MM, et al. Mechanism of the interaction between circular RNA KRT17 and miR-485$5 \mathrm{p}$ to regulate $\mathrm{Wnt} / \beta$-catenin signaling pathway and affect the progression of gastric cancer. Journal of Clinical and Experimental Medicine 2020;19:565-70.

30. $\mathrm{Xu} \mathrm{G}$. The functional roles and clinical significance of circCCDC66 in gastric cancer [Master]. Jiangsu University, 2019.

31. Xie JB, Wang SJ, Guo RS, et al. Effects of circular RNA VANGL1 on proliferation and apoptosis of gastric cancer cells. Journal of Tongji University (Medical Science) 2019;40:281-7.

32. Lu J, Wang YH, Yoon C, et al. Circular RNA circRanGAP1 regulates VEGFA expression by targeting miR$877-3$ p to facilitate gastric cancer invasion and metastasis. Cancer Lett 2020;471:38-48.

33. Liu $W$, Gao $M$, Liu $W$, et al. Preliminary study on the effect of hsa_circ_0008797 on cisplatin resistance in gastric cancer cells. Acta Universitatis Medicinalis Anhui 2019;54:1702-7.

34. Li Q, Geng S, Yuan H, et al. Circular RNA expression profiles in extracellular vesicles from the plasma of patients with pancreatic ductal adenocarcinoma. FEBS Open Bio 2019;9:2052-62.

35. Guo X, Zhou Q, Su D, et al. Circular RNA circBFAR promotes the progression of pancreatic ductal adenocarcinoma via the miR-34b-5p/MET/Akt axis. Mol Cancer 2020;19:83.

36. Liu Y, Xia L, Dong L, et al. CircHIPK3 Promotes Gemcitabine (GEM) Resistance in Pancreatic Cancer Cells by Sponging miR-330-5p and Targets RASSF1. Cancer Manag Res 2020;12:921-9.

37. Yang CM, Qiao GL, Song LN, et al. Clinical value of plasma has_circ_0003998 in diagnosis and prognostic evaluation of primary hepatocellular carcinoma. Chinese Journal of Laboratory Diagnosis 2019;23:761-6.

38. Li Y, Kong M, Zhu B, et al. Expression of hsa_ circ_0128298 in hepatocellular carcinoma and its functional roles in tumor growth. Chinese Journal of Clinical Laboratory Science 2019;37:568-73.

39. Fu Y, Cai L, Lei X, et al. Circular RNA ABCB10 promotes hepatocellular carcinoma progression by increasing HMG20A expression by sponging miR-670-3p. Cancer Cell Int 2019;19:338. Retraction in: Cancer Cell Int 2020;20:485.
40. Yu K, Li Q, Wang W, et al. Circular RNA RNF13 promotes proliferation of colorectal cancer cells by regulating miR-196b-5p expression. Tumor 2019;39:887-96.

41. Wang H, Wang L, Zhang Y, et al. The expression and clinical significance of plasma circPRMT5 in colorectal cancer patients. Chinese Journal of Colorectal Diseases (Electronic Edition) 2019;8:390-4.

42. Zheng X, Chen L, Zhou Y, et al. A novel protein encoded by a circular RNA circPPP1R12A promotes tumor pathogenesis and metastasis of colon cancer via HippoYAP signaling. Mol Cancer 2019;18:47.

43. Chen C, Huang Z, Mo X, et al. The circular RNA 001971/ miR-29c-3p axis modulates colorectal cancer growth, metastasis, and angiogenesis through VEGFA. J Exp Clin Cancer Res 2020;39:91.

44. Gao Min, Liu Wenbo, Zhao Qihong, et al. Effects of hsa_circ_0010985 on the proliferation and apoptosis of gastric cancer cells. Acta Universitatis Medicinalis Anhui 2019;54:1387-91.

45. Wu L, Liu D, Yang Y. Enhanced expression of circular RNA circ-DCAF6 predicts adverse prognosis and promotes cell progression via sponging miR-1231 and miR-1256 in gastric cancer. Exp Mol Pathol 2019;110:104273.

46. Yu X, Ding H, Yang L, et al. Reduced expression of circRNA hsa_circ_0067582 in human gastric cancer and its potential diagnostic values. J Clin Lab Anal 2020;34:e23080.

47. Liang M, Huang G, Liu Z, et al. Elevated levels of hsa circ_006100 in gastric cancer promote cell growth and metastasis via miR-195/GPRC5A signalling. Cell Prolif 2019;52:e12661.

48. Luo Z, Rong Z, Zhang J, et al. Circular RNA circCCDC9 acts as a miR-6792-3p sponge to suppress the progression of gastric cancer through regulating CAV1 expression. Mol Cancer 2020;19:86.

49. Chen Y, Li Z, Zhang M, et al. Circ-ASH2L promotes tumor progression by sponging miR-34a to regulate Notch1 in pancreatic ductal adenocarcinoma. J Exp Clin Cancer Res 2019;38:466.

Cite this article as: Wang H, Xiang Y, Hu R, Ji R, Wang Y. Research progress of circular RNA in digestive tract tumors: a narrative review. Transl Cancer Res 2020;9(12):7632-7641. doi: $10.21037 /$ tcr-20-2708 